\title{
Thermal Conductivity, Electrical Resistivity, and Thermopower of Aerospace Alloys from 4 to $300 \mathrm{~K}^{*}$
}

\author{
J. G. Hust, $* *$ D. H. Weitzel, $* *$ and R. L. Powell** \\ Institute for Basic Standards, National Bureau of Standards, Boulder, Colorado 80302
}

(March 10, 1971)

\begin{abstract}
Thermal conductivity, electrical resistivity, and thermopower have been measured for several aerospace alloys: titanium alloy A110-AT, aluminum alloy 7039, Inconel 718, and Hastelloy X. Tables and graphs of the measured properties and Lorenz ratio are presented over the range 4 to $300 \mathrm{~K}$. Comparisons to other measurements and theoretical analysis of the data are included. The uncertainties of the property data are estimated as (1) 0.7 to 2.5 percent for thermal conductivity, (2) 0.25 percent in electrical resistivity, and (3) about $0.1 \mu \mathrm{V} / \mathrm{K}$ in thermopower.
\end{abstract}

Key words: Aluminum alloy; cryogenics; electrical resistivity; Lorenz ratio; nickel alloys; Seebeck effect; thermal conductivity; titanium alloy; transport properties.

\section{Introduction}

The development of new structural materials and renewed interest in existing materials by the aerospace industry is creating a demand for thermal and electrical property measurements on these materials. Such data are needed for the selection of suitable construction materials and the prediction of operating characteristics of low temperature systems. To help satisfy the immediate needs for these data, an apparatus has been built to measure the thermal conductivity, electrical resistivity, and thermopower of solids. This apparatus is designed to measure samples with thermal conductivities varying from 0.1 to $5,000 \mathrm{~W} \mathrm{~m}^{-1} \mathrm{~K}^{-1}$ at temperatures from 4 to $300 \mathrm{~K}$.

Thermal conductivity data of technically important solids accurate to 5 percent satisfy current demands. However, future demands will likely be more stringent. For this reason this program is directed toward the acquisition of thermal conductivity data accurate to within 1 percent; this is difficult to do especially for poor conductors and temperatures above about $120 \mathrm{~K}$, because of the difficulty of maintaining thermal losses at a sufficiently low level.

This paper contains results of measurements on titanium A-110 AT, Iconel $718,{ }^{1}$ Hastelloy $\mathrm{X}$, and aluminum 7039.

\section{Apparatus}

The present apparatus is similar to that described by Powell, et al. [1].2 A detailed description of the present

*This work was carried out at the National Bureau of Standards under the sponsorship of the NASA (SNPO-C) Contract R-45.

${ }^{* *}$ Cryogenics Division, National Bureau of Standards. Boulder, Colorado 80302.

${ }^{1}$ The use in this paper of trade names of specific products is essential to a proper understanding of the work presented. Their use in no way implies any approval, endorsement, or recommendation by NBS. (See reference [8]).

${ }^{2}$ Figures in brackets indicate the literature references at the end of this paper. system is given by Hust et al. [2]; therefore, only' a brief description of this apparatus is given here. Figure 1 illustrates the cryostat of this system.

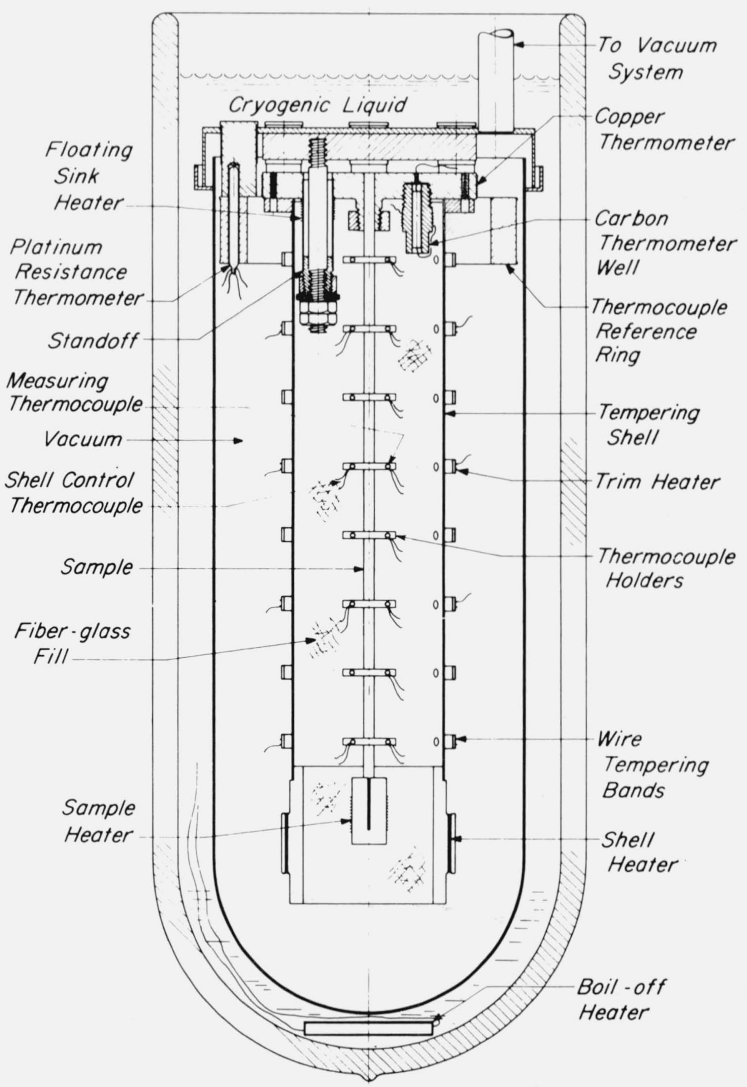

FIGURE 1. Thermal conductivity apparatus. 
The apparatus used is based on the axial one-dimensional heat flow method. The specimen is a cylindrical rod with a heater at one end and temperature controlled heat sink at the other. The heat sink is controlled to any temperature from 4 to $300 \mathrm{~K}$ by means of several cryogenic baths and an automatic electronic temperature controller. The temperature distribution along the specimen is measured with respect to a constant temperature reference block with eight Chromel [8] versus $\mathrm{Au}-\mathrm{Fe}(\mathrm{Au}-0.07$ at. \% Fe) thermocouples. The reference block temperature is determined with a platinum resistance thermometer at temperatures above $10 \mathrm{~K}$ and from the vapor pressure of liquid helium near $4 \mathrm{~K}$. The thermocouple calibrations are based on standard reference tables by Sparks, et al. (NBS Monograph - in review) and small corrections to these tables based on spool calibrations at $4 \mathrm{~K}, 20 \mathrm{~K}, 78 \mathrm{~K}$, and $273 \mathrm{~K}$ and in-place calibrations from 4 to $30 \mathrm{~K}$. The platinum resistance thermometer was calibrated by NBS, Washington. Details of these calibrations are given by Hust et al. [2]. The sample assembly is surrounded by a cylindrical temperaturecontrolled shield to reduce heat losses by conduction and radiation. To further reduce losses by radiation, the space between the shield and the specimen is filled with high-density glass fibers. The entire sampleshield assembly is enclosed in a container and evacuated to less than $10^{-5}$ torr $\left(1.3 \times 10^{-3} \mathrm{~N} / \mathrm{m}^{2}\right)$.

\section{Specimen Preparation and Measurement Techniques}

The specimens are machined and ground to specified nominal dimensions, after which they are accurately measured in a temperature-controlled measurement lab. Without further undue mechanical or thermal abuse, each specimen is fitted with thermocouple holders and heater. The specimen assembly is installed in the cryostat, the space between the shell and specimen is packed with glass fiber, and the vecuum can is soldered into place. The cryostat is evacuated to better than $10^{-5}$ torr and is subsequently cooled with the desired cryogenic liquid. The specimen is brought into equilibrium with the bath temperature and the emf of each thermocouple at zero temperature gradient is read. These zero corrections, caused by various inhomogenities in the circuit, are considered to be constant for all runs with each cryogenic bath.

Data on a given run are taken only after thermal steady state has been established with a vacuum of better than $10^{-5}$ torr. Thermal steady state is considered established after systematic drift of the indicated thermocouple temperatures are below the detection or control limit of approximately 1 millidegree per hour.

Isothermal electrical resistivity data are taken at the same time that the zero emfs are recorded. Also, to obtain further isothermal resistivity data and information regarding the differences between the eight measuring thermocouples, data are taken with the floating sink above the temperature of the surrounding bath but with no heat input to the specimen. The thermocouples thus indicate the temperature difference from the specimen to the reference block. If the specimen is in equilibrium with the floating sink, then all eight thermocouples should produce the same emf. The scatter in these recorded emfs is an indication of the validity of using a single calibration table for all eight thermocouples. No significant deviations between thermocouples have been detected by this procedure.

\section{Results}

Measurements have been performed on several aerospace alloys. The characterization data for these specimens are presented in table 1 . The experimental data were represented by mathematical functions. The functions chosen to represent thermal conductivity, electrical resistivity, and thermopower are rather arbitrary, since adequate relationships based upon theoretical considerations are not available. These functional forms are given by eqs (1), (2), and (3) respectively:

$$
\ln \lambda=\sum_{i=1}^{n} a_{i}[\ln T]^{i+1}
$$

TABLE 1. Specimen characterization

\begin{tabular}{|c|c|c|c|c|}
\hline $\begin{array}{c}\text { Material } \\
\text { (diameter) }\end{array}$ & $\begin{array}{l}\text { Condition } \\
\text { (structure) }\end{array}$ & $\begin{array}{l}\text { Rockwell } \\
\text { Hardness }\end{array}$ & $\begin{array}{l}\text { Av. Grain } \\
\text { diameter }\end{array}$ & $\begin{array}{l}\text { Composition weight percent } \\
\text { (less than } 0.1 \% \text {-listed only) }\end{array}$ \\
\hline $\begin{array}{l}\text { Ti-A110 AT } \\
(1.13 \mathrm{~cm})\end{array}$ & $\begin{array}{l}\text { Annealed } \\
(\text { HCP) }\end{array}$ & $\mathrm{C}-35$ & $0.015 \mathrm{~mm}$ & Ti-91.5, Al-5.5, Sn-2.5, Fe-0.2, C, N, H. \\
\hline $\begin{array}{c}\mathrm{Al} 7039 \\
(0.367 \mathrm{~cm})\end{array}$ & $\begin{array}{l}\text { T } 61 \\
\text { (FCC) }\end{array}$ & $\mathrm{B}-75$ & $\begin{array}{l}0.005 \mathrm{~mm} \text { (elongation along } \\
\text { sample axis to } 0.05 \mathrm{~mm} \text { ) }\end{array}$ & $\begin{array}{l}\text { Al-93.0, Zn-3.6, Mg-2.55, Mn-0.23, Cr-0.20, Fe, Cu, } \\
\text { Si, Ti, Be. }\end{array}$ \\
\hline $\begin{array}{l}\text { Inconel } 718 \\
(1.13 \mathrm{~cm})\end{array}$ & $\begin{array}{l}\text { Age-hardened } \\
\qquad(\text { BCC + FCC ppt })^{\text {a }}\end{array}$ & $\mathrm{C}-39$ & $0.06 \mathrm{~mm}$ & $\begin{array}{l}\text { Ni-54.57, Cr-18.06, Fe-17.08, Nb+ Ta-5.12, Mo-3.18, } \\
\text { Ti-0.85, Al-0.44, Mn-0.29, Si-0.24, Cu, C, S. }\end{array}$ \\
\hline$\underset{(1.13 \mathrm{~cm})}{\text { Hastelloy X }}$ & $\begin{array}{l}\text { Annealed }(\mathrm{BCC}+\mathrm{FCC} \\
{\text { ppt })^{\mathrm{a}}}^{\mathrm{a}}\end{array}$ & $\mathrm{B}-88$ & $0.08 \mathrm{~mm}$ & $\begin{array}{l}\text { Ni-49.0, Cr-21.06, Fe-17.58, Mo-9.15, Co-1.45, } \\
\text { W-0.65, Mn-0.53, Si-0.43, C-0.12, P, S. }\end{array}$ \\
\hline
\end{tabular}

${ }^{\mathrm{a}} \mathrm{ppt}=$ precipitate. 


$$
\begin{gathered}
\rho=\sum_{i=1}^{m} b_{i}[\ln T]^{i+1} \\
S=\sum_{i=1}^{1} c_{i}\left[\ln T^{\prime}\right]^{i} / T^{\prime} ; T^{\prime}=\frac{T}{10}+1
\end{gathered}
$$

where $\lambda=$ thermal conductivity, $\rho=$ electrical resistivity, $S=$ thermopower, and $T=$ temperature in SI Units.

The parameters, determined by least squares fits, are tabulated in tables 2 through 5 . The number of significant figures retained in these parameters is dictated by the number of terms in eqs (1), (2), and (3), and only indirectly by the accuracy of the data. Values of thermal conductivity, electrical resistivity, absolute thermopower, and Lorenz ratio as computed from eqs (1), (2), and (3) are tabulated in tables 6 through 9 and illustrated in figures 2 through 7 . The raw experimental data have been tabulated in an informal report for future reference.

A detailed error analysis for these measurements has been presented previously by Hust et al. [2]. Based on this analysis of systematic and random

TABle 2. Parameters in eqs (1), (2), and (3) for Ti All0-AT

\begin{tabular}{cccc}
\hline \hline $\mathrm{i}$ & Thermal conductivity & Electrical resistivity & \multicolumn{1}{c}{ Thermopower } \\
\hline 1 & -5.06395097 & $7.89252702 \times 10^{-7}$ & $-1.58121391 \times 10$ \\
2 & 6.88712311 & $1.49763681 \times 10^{-6}$ & $3.79180055 \times 10$ \\
3 & -3.90357743 & $-1.59177332 \times 10^{-6}$ & $-8.41032221 \times 10$ \\
4 & 1.19805789 & $8.96843641 \times 10^{-7}$ & $9.74696929 \times 10$ \\
5 & $-2.09020637 \times 10^{-1}$ & $-2.88887717 \times 10^{-7}$ & $-1.12974481 \times 10^{2}$ \\
6 & $1.95307646 \times 10^{-2}$ & $5.30624488 \times 10^{-8}$ & $6.18987000 \times 10$ \\
7 & $-7.59426352 \times 10^{-4}$ & $-5.14188674 \times 10^{-9}$ & $-1.54216675 \times 10$ \\
8 & & $2.04423026 \times 10^{-10}$ & 1.35460142 \\
\hline
\end{tabular}

TABLE 3. Parameters in eqs (1), (2), and (3) for Al 7039

\begin{tabular}{ccrr}
\hline \hline $\mathrm{i}$ & Thermal conductivity & Electrical resistivity & \multicolumn{1}{c}{ Thermopower } \\
\hline 1 & 2.29632521 & $1.28748725 \times 10^{-7}$ & $1.71060776 \times 10$ \\
2 & -1.54727504 & $-3.27608149 \times 10^{-7}$ & $-1.33360752 \times 10^{2}$ \\
3 & $4.00341352 \times 10^{-1}$ & $4.07400149 \times 10^{-7}$ & $3.11404848 \times 10^{2}$ \\
4 & $2.88936392 \times 10^{-3}$ & $-2.79922547 \times 10^{-7}$ & $-3.37068033 \times 10^{2}$ \\
5 & $-2.15351278 \times 10^{-2}$ & $1.16239500 \times 10^{-7}$ & $1.57586179 \times 10^{2}$ \\
6 & $3.88045930 \times 10^{-3}$ & $-2.98620355 \times 10^{-8}$ & $-3.31898139 \times 10$ \\
7 & $-2.18079207 \times 10^{-4}$ & $4.63166504 \times 10^{-9}$ & 2.49163872 \\
8 & & $-3.96374984 \times 10^{-10}$ & \\
9 & & $1.43605591 \times 10^{-11}$ & \\
\hline
\end{tabular}

TABle 4. Parameters in eqs (1), (2), and (3) for Inconel 718

\begin{tabular}{ccrr}
\hline \hline $\mathrm{i}$ & Thermal conductivity & Electrical resistivity & \multicolumn{1}{c}{ Thermopower } \\
\hline 1 & -5.46241719 & $1.12285087 \times 10^{-6}$ & $5.48360101 \times 10$ \\
2 & 7.39689278 & $-7.83810447 \times 10^{-8}$ & $-3.01984465 \times 10^{2}$ \\
3 & -4.16174867 & $5.63890447 \times 10^{-8}$ & $6.85469757 \times 10^{2}$ \\
4 & 1.26896416 & $-1.93288570 \times 10^{-8}$ & $-7.68205488 \times 10^{2}$ \\
5 & $-2.20152847 \times 10^{-1}$ & $2.96162062 \times 10^{-9}$ & $4.56243644 \times 10^{2}$ \\
6 & $2.04649099 \times 10^{-2}$ & $-1.50481839 \times 10^{-10}$ & $-1.47829752 \times 10^{2}$ \\
7 & $-7.91806469 \times 10^{-4}$ & & $2.48887223 \times 10$ \\
8 & & & -1.70855972 \\
\hline
\end{tabular}

TABle 5. Parameters in eqs (1), (2), and (3) for Hastelloy $X$

\begin{tabular}{cccr}
\hline \hline $\mathrm{i}$ & Thermal conductivity & Electrical resistivity & \multicolumn{1}{c}{ Thermopower } \\
\hline 1 & -4.55464242 & $1.08125768 \times 10^{-6}$ & $3.20187561 \times 10$ \\
2 & 6.50507423 & $2.50203640 \times 10^{-8}$ & $-2.06088289 \times 10^{2}$ \\
3 & -3.79427905 & $-2.37968596 \times 10^{-8}$ & $4.88192143 \times 10^{2}$ \\
4 & 1.18719162 & $1.08737546 \times 10^{-8}$ & $-5.64341813 \times 10^{2}$ \\
5 & $-2.09767231 \times 10^{-1}$ & $-2.85718690 \times 10^{-9}$ & $3.35752457 \times 10^{2}$ \\
6 & $1.97476492 \times 10^{-2}$ & $4.00630494 \times 10^{-10}$ & $-1.07878568 \times 10^{2}$ \\
7 & $-7.70496356 \times 10^{-4}$ & $-2.06631014 \times 10^{-11}$ & $1.79312534 \times 10$ \\
8 & & & -1.21659772 \\
\hline
\end{tabular}


TABLE 6. Transport properties of Ti Al10-AT

\begin{tabular}{|c|c|c|c|c|}
\hline $\begin{array}{l}\text { Temp } \\
(\mathrm{K})\end{array}$ & $\begin{array}{c}\text { Thermal } \\
\text { conductivity } \\
\left(\mathbf{W m}^{-1} \mathrm{~K}^{-1}\right)\end{array}$ & $\begin{array}{l}\text { Electrical } \\
\text { resistivity } \\
(\mu \text { ohm m) }\end{array}$ & $\begin{array}{l}\text { Lorenz } \\
\text { ratio } \times 10^{8} \\
\left(\mathrm{~V}^{2} / \mathrm{K}^{2}\right)\end{array}$ & $\begin{array}{c}\text { Thermopower } \\
(\mu \mathrm{V} / \mathrm{K})\end{array}$ \\
\hline $\begin{array}{r}6 \\
7 \\
8 \\
9 \\
10\end{array}$ & $\begin{array}{r}0.555 \\
.662 \\
.773 \\
.885 \\
.996\end{array}$ & $\begin{array}{l}1.366 \\
1.365 \\
1.365 \\
1.365 \\
1.365\end{array}$ & $\begin{array}{l}12.6 \\
12.9 \\
13.2 \\
13.4 \\
13.6\end{array}$ & $\begin{array}{r}-0.30 \\
-.35 \\
-.39 \\
-.45 \\
-.50\end{array}$ \\
\hline $\begin{array}{l}12 \\
14 \\
16 \\
18 \\
20\end{array}$ & $\begin{array}{l}1.21 \\
1.41 \\
1.60 \\
1.78 \\
1.95\end{array}$ & $\begin{array}{l}1.365 \\
1.364 \\
1.364 \\
1.364 \\
1.364\end{array}$ & $\begin{array}{l}13.8 \\
13.8 \\
13.7 \\
13.5 \\
13.3\end{array}$ & $\begin{array}{r}-.58 \\
-.67 \\
-.78 \\
-.89 \\
-1.03\end{array}$ \\
\hline $\begin{array}{l}25 \\
30 \\
35 \\
40 \\
45\end{array}$ & $\begin{array}{l}2.32 \\
2.64 \\
2.92 \\
3.17 \\
3.39\end{array}$ & $\begin{array}{l}1.364 \\
1.364 \\
1.366 \\
1.368 \\
1.371\end{array}$ & $\begin{array}{l}12.7 \\
12.0 \\
11.4 \\
10.8 \\
10.3\end{array}$ & $\begin{array}{l}-1.38 \\
-1.72 \\
-2.03 \\
-2.30 \\
-2.54\end{array}$ \\
\hline $\begin{array}{l}50 \\
55 \\
60 \\
65 \\
70\end{array}$ & $\begin{array}{l}3.59 \\
3.76 \\
3.92 \\
4.07 \\
4.20\end{array}$ & $\begin{array}{l}1.375 \\
1.379 \\
1.383 \\
1.388 \\
1.393\end{array}$ & $\begin{array}{l}9.86 \\
9.43 \\
9.04 \\
8.69 \\
8.36\end{array}$ & $\begin{array}{l}-2.76 \\
-2.95 \\
-3.11 \\
-3.26 \\
-3.39\end{array}$ \\
\hline $\begin{array}{l}75 \\
80 \\
85 \\
90 \\
95\end{array}$ & $\begin{array}{l}4.32 \\
4.44 \\
4.54 \\
4.64 \\
4.74\end{array}$ & $\begin{array}{l}1.398 \\
1.404 \\
1.409 \\
1.415 \\
1.421\end{array}$ & $\begin{array}{l}8.06 \\
7.78 \\
7.53 \\
7.30 \\
7.08\end{array}$ & $\begin{array}{l}-3.51 \\
-3.62 \\
-3.72 \\
-3.82 \\
-3.91\end{array}$ \\
\hline $\begin{array}{l}100 \\
110 \\
120 \\
130 \\
140\end{array}$ & $\begin{array}{l}4.83 \\
5.00 \\
5.17 \\
5.33 \\
5.50\end{array}$ & $\begin{array}{l}1.426 \\
1.438 \\
1.450 \\
1.461 \\
1.473\end{array}$ & $\begin{array}{l}6.89 \\
6.54 \\
6.25 \\
6.00 \\
5.78\end{array}$ & $\begin{array}{l}-3.99 \\
-4.16 \\
-4.31 \\
-4.46 \\
-4.61\end{array}$ \\
\hline $\begin{array}{l}150 \\
160 \\
170 \\
180 \\
190\end{array}$ & $\begin{array}{l}5.66 \\
5.83 \\
6.00 \\
6.17 \\
6.35\end{array}$ & $\begin{array}{l}1.484 \\
1.495 \\
1.506 \\
1.517 \\
1.528\end{array}$ & $\begin{array}{l}5.60 \\
5.45 \\
5.32 \\
5.20 \\
5.11\end{array}$ & $\begin{array}{l}-4.77 \\
-4.92 \\
-5.07 \\
-5.22 \\
-5.38\end{array}$ \\
\hline $\begin{array}{l}200 \\
220 \\
240 \\
260 \\
280\end{array}$ & $\begin{array}{l}6.53 \\
6.89 \\
7.24 \\
7.58 \\
7.90\end{array}$ & $\begin{array}{l}1.538 \\
1.559 \\
1.579 \\
1.598 \\
1.617\end{array}$ & $\begin{array}{l}5.02 \\
4.88 \\
4.76 \\
4.66 \\
4.56\end{array}$ & $\begin{array}{l}-5.53 \\
-5.83 \\
-6.12 \\
-6.40 \\
-6.66\end{array}$ \\
\hline 300 & 8.17 & 1.636 & 4.45 & -6.90 \\
\hline
\end{tabular}

TABLE 7. Transport properties of $\mathrm{Al} 7039$

\begin{tabular}{|c|c|c|c|c|}
\hline$\underset{(\mathrm{K})}{\text { Temp }}$ & $\begin{array}{c}\text { Thermal } \\
\text { conductivity } \\
\left(\mathbf{W m}^{-1} \mathrm{~K}^{-1}\right)\end{array}$ & $\begin{array}{l}\text { Electrical } \\
\text { resistivity } \\
(\mu \text { ohm m) }\end{array}$ & $\begin{array}{c}\text { Lorenz } \\
\text { ratio } \times 10^{8} \\
\left(\mathbf{V}^{2} / \mathrm{K}^{2}\right)\end{array}$ & $\begin{array}{c}\text { Thermopower } \\
(\mu \mathrm{V} / \mathrm{K})\end{array}$ \\
\hline 6 & 8.6 & 0.01734 & 2.47 & -0.13 \\
\hline 7 & 10.1 & .01736 & 2.50 & -0.15 \\
\hline 8 & 11.6 & .01737 & 2.51 & -0.17 \\
\hline 9 & 13.1 & .01736 & 2.52 & -0.19 \\
\hline 10 & 14.5 & .01735 & 2.52 & -0.20 \\
\hline 12 & 17.4 & .01734 & 2.52 & -0.21 \\
\hline 14 & 20.4 & .01734 & 2.52 & -0.23 \\
\hline 16 & 23.3 & .01735 & 2.53 & -0.27 \\
\hline 18 & 26.2 & .01736 & 2.53 & -0.33 \\
\hline 20 & 29.2 & .01737 & 2.53 & -0.41 \\
\hline 25 & 36.3 & .01742 & 2.53 & -0.65 \\
\hline 30 & 43.1 & .01748 & 2.51 & -0.88 \\
\hline 35 & 49.5 & .01759 & 2.49 & -1.09 \\
\hline 40 & 55.3 & .01775 & 2.45 & -1.26 \\
\hline 45 & 60.6 & .01795 & 2.42 & -1.41 \\
\hline 50 & 65.4 & .01821 & 2.38 & -1.53 \\
\hline 55 & 69.7 & .01852 & 2.35 & -1.62 \\
\hline 60 & 73.6 & .01887 & 2.31 & -1.69 \\
\hline 65 & 77.1 & .01926 & 2.29 & -1.74 \\
\hline 70 & 80.3 & .01970 & 2.26 & -1.78 \\
\hline 75 & 83.3 & .02016 & 2.24 & -1.81 \\
\hline 80 & 86.0 & .02065 & 2.22 & -1.82 \\
\hline 85 & 88.5 & .02117 & 2.21 & -1.83 \\
\hline 90 & 90.9 & .02171 & 2.19 & -1.83 \\
\hline 95 & 93.1 & .02227 & 2.18 & -1.83 \\
\hline 100 & 95.2 & .02284 & 2.18 & -1.82 \\
\hline 110 & 99.2 & .02402 & 2.17 & -1.80 \\
\hline 120 & 103. & .02524 & 2.17 & -1.78 \\
\hline 130 & 107. & .02648 & 2.17 & -1.75 \\
\hline 140 & 110. & .02773 & 2.18 & -1.72 \\
\hline 150 & 114. & .02898 & 2.20 & -1.70 \\
\hline 160 & 117. & .03024 & 2.21 & -1.68 \\
\hline 170 & 121. & .03149 & 2.24 & -1.66 \\
\hline 180 & 124. & .03274 & 2.26 & -1.65 \\
\hline 190 & 128. & .03398 & 2.28 & -1.64 \\
\hline 200 & 131. & .03522 & 2.31 & -1.63 \\
\hline 220 & 138. & .03768 & 2.36 & -1.62 \\
\hline 240 & 144. & .04012 & 2.40 & -1.62 \\
\hline 260 & 149. & .04255 & 2.44 & -1.62 \\
\hline 280 & 154. & .04499 & 2.47 & -1.63 \\
\hline
\end{tabular}


TABLE 8. Transport properties of Inconel 718

\begin{tabular}{|c|c|c|c|c|}
\hline $\begin{array}{c}\text { Temp } \\
\text { (K) }\end{array}$ & $\begin{array}{c}\text { Thermal } \\
\text { conductivity } \\
\left(\mathrm{Wm}^{-1} \mathrm{~K}^{-1}\right)\end{array}$ & $\begin{array}{l}\text { Electrical } \\
\text { resistivity } \\
(\mu \mathrm{ohm} \mathrm{m})\end{array}$ & $\begin{array}{c}\text { Lorenz } \\
\text { ratio } \times 10^{8} \\
\left(\mathrm{~V}^{2} / \mathrm{K}^{2}\right)\end{array}$ & $\begin{array}{c}\text { Thermopower } \\
(\mu \mathrm{V} / \mathrm{K})\end{array}$ \\
\hline 7 & 0.670 & 1.080 & 10.3 & 0.12 \\
\hline 8 & .801 & 1.079 & 10.8 & .12 \\
\hline 9 & .938 & 1.079 & 11.2 & .13 \\
\hline 10 & 1.08 & 1.079 & 11.6 & .14 \\
\hline 12 & 1.36 & 1.078 & 12.2 & .20 \\
\hline 14 & 1.63 & 1.078 & 12.6 & .26 \\
\hline 16 & 1.90 & 1.077 & 12.8 & .30 \\
\hline 18 & 2.16 & 1.077 & 12.9 & .32 \\
\hline 20 & 2.40 & 1.077 & 12.9 & .32 \\
\hline 25 & 2.97 & 1.076 & 12.8 & .28 \\
\hline 30 & 3.48 & 1.076 & 12.5 & .24 \\
\hline 35 & 3.94 & 1.076 & 12.1 & .21 \\
\hline 40 & 4.35 & 1.076 & 11.7 & .19 \\
\hline 45 & 4.72 & 1.077 & 11.3 & .19 \\
\hline 50 & 5.05 & 1.078 & 10.9 & .18 \\
\hline 55 & 5.35 & 1.079 & 10.5 & .19 \\
\hline 60 & 5.62 & 1.080 & 10.1 & .21 \\
\hline 65 & 5.86 & 1.081 & 9.75 & .23 \\
\hline 70 & 6.09 & 1.082 & 9.41 & .26 \\
\hline 75 & 6.29 & 1.083 & 9.08 & .29 \\
\hline 80 & 6.47 & 1.085 & 8.77 & .32 \\
\hline 85 & 6.64 & 1.086 & 8.49 & .35 \\
\hline 90 & 6.80 & 1.087 & 8.22 & .38 \\
\hline 95 & 6.95 & 1.089 & 7.96 & .42 \\
\hline 100 & 7.09 & 1.090 & 7.73 & .45 \\
\hline 110 & 7.34 & 1.093 & 7.30 & .52 \\
\hline 120 & 7.58 & 1.096 & 6.93 & .60 \\
\hline 130 & 7.81 & 1.100 & 6.60 & .67 \\
\hline 140 & 8.02 & 1.103 & 6.32 & .74 \\
\hline 150 & 8.24 & 1.106 & 6.07 & .81 \\
\hline 160 & 8.45 & 1.109 & 5.86 & .88 \\
\hline 170 & 8.66 & 1.112 & 5.67 & .95 \\
\hline 180 & 8.88 & 1.115 & 5.50 & 1.02 \\
\hline 190 & 9.09 & 1.118 & 5.35 & 1.08 \\
\hline 200 & 9.30 & 1.121 & 5.22 & 1.15 \\
\hline 220 & 9.73 & 1.127 & 4.98 & 1.27 \\
\hline 240 & 10.1 & 1.133 & 4.79 & 1.39 \\
\hline 260 & 10.5 & 1.139 & 4.61 & 1.51 \\
\hline 280 & 10.9 & 1.144 & 4.44 & 1.62 \\
\hline
\end{tabular}

TABLE 9. Transport properties of Hastelloy $X$

\begin{tabular}{|c|c|c|c|c|}
\hline $\begin{array}{c}\text { Temp } \\
\text { (K) }\end{array}$ & $\begin{array}{c}\text { Thermal } \\
\text { conductivity } \\
\left(\mathrm{Wm}^{-1} \mathrm{~K}^{-1}\right)\end{array}$ & $\begin{array}{l}\text { Electrical } \\
\text { resistivity } \\
(\mu \text { ohm m) }\end{array}$ & $\begin{array}{c}\text { Lorenz } \\
\text { ratio } \times 10^{8} \\
\left(\mathrm{~V}^{2} / \mathrm{K}^{2}\right)\end{array}$ & $\begin{array}{c}\text { Thermopower } \\
(\mu \mathrm{V} / \mathrm{K})\end{array}$ \\
\hline 7 & 0.946 & 1.089 & 14.7 & -0.04 \\
\hline 8 & 1.11 & 1.089 & 15.1 & -.04 \\
\hline 9 & 1.27 & 1.088 & 15.3 & -.05 \\
\hline 10 & 1.43 & 1.088 & 15.5 & -.04 \\
\hline 12 & 1.73 & 1.087 & 15.7 & -.01 \\
\hline 14 & 2.01 & 1.087 & 15.6 & .02 \\
\hline 16 & 2.27 & 1.087 & 15.4 & .04 \\
\hline 18 & 2.51 & 1.087 & 15.1 & .04 \\
\hline 20 & 2.73 & 1.087 & 14.8 & .02 \\
\hline 25 & 3.23 & 1.087 & 14.1 & -.06 \\
\hline 30 & 3.67 & 1.087 & 13.3 & -.14 \\
\hline 35 & 4.05 & 1.087 & 12.6 & -.20 \\
\hline 40 & 4.38 & 1.088 & 11.9 & -.25 \\
\hline 45 & 4.68 & 1.089 & 11.3 & -.29 \\
\hline 50 & 4.95 & 1.090 & 10.8 & -.32 \\
\hline 55 & 5.19 & 1.091 & 10.3 & -.33 \\
\hline 60 & 5.40 & 1.092 & 9.83 & -.34 \\
\hline 65 & 5.59 & 1.093 & 9.41 & -.34 \\
\hline 70 & 5.77 & 1.094 & 9.02 & -.33 \\
\hline 75 & 5.93 & 1.095 & 8.66 & -.32 \\
\hline 80 & 6.07 & 1.096 & 8.32 & -.31 \\
\hline 85 & 6.21 & 1.098 & 8.02 & -.29 \\
\hline 90 & 6.33 & 1.099 & 7.73 & -.27 \\
\hline 95 & 6.45 & 1.100 & 7.47 & -.25 \\
\hline 100 & 6.56 & 1.101 & 7.23 & -.22 \\
\hline 110 & 6.77 & 1.104 & 6.79 & -.18 \\
\hline 120 & 6.96 & 1.107 & 6.42 & -.12 \\
\hline 130 & 7.15 & 1.209 & 6.10 & -.06 \\
\hline 140 & 7.34 & 1.112 & 5.83 & -.00 \\
\hline 150 & 7.53 & 1.114 & 5.59 & .05 \\
\hline 160 & 7.72 & 1.117 & 5.39 & .11 \\
\hline 170 & 7.91 & 1.120 & 5.21 & .16 \\
\hline 180 & 8.11 & 1.122 & 5.05 & .22 \\
\hline 190 & 8.31 & 1.125 & 4.92 & .28 \\
\hline 200 & 8.51 & 1.127 & 4.80 & .33 \\
\hline 220 & 8.93 & 1.132 & 4.59 & .44 \\
\hline 240 & 9.34 & 1.137 & 4.42 & .54 \\
\hline 260 & 9.74 & 1.142 & 4.28 & .64 \\
\hline 280 & 10.1 & 1.146 & 4.14 & .74 \\
\hline 300 & 10.4 & 1.151 & 4.01 & .84 \\
\hline
\end{tabular}




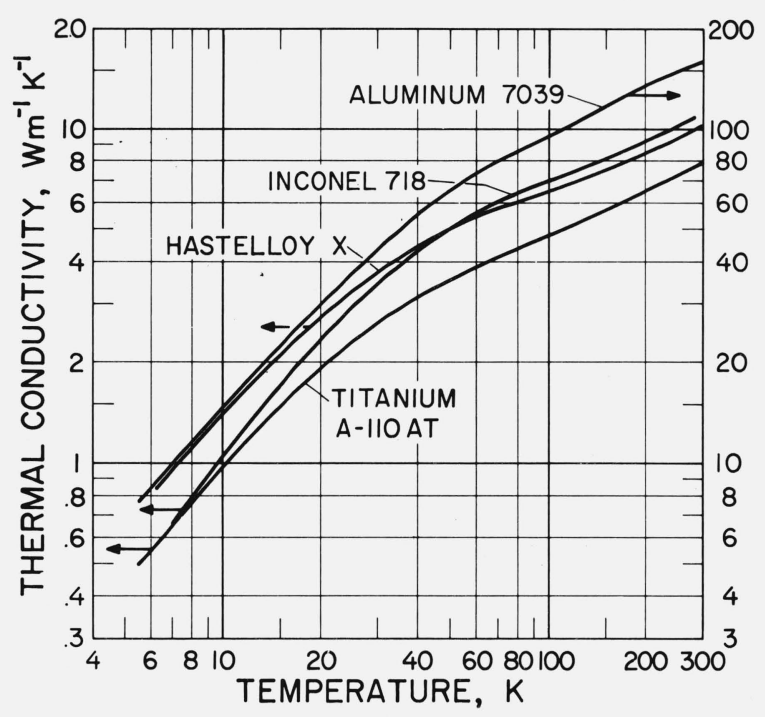

FIgURE 2. Thermal conductivity of titanium A110-AT, aluminum 7039, Inconel 718, and Hastelloy $X$.

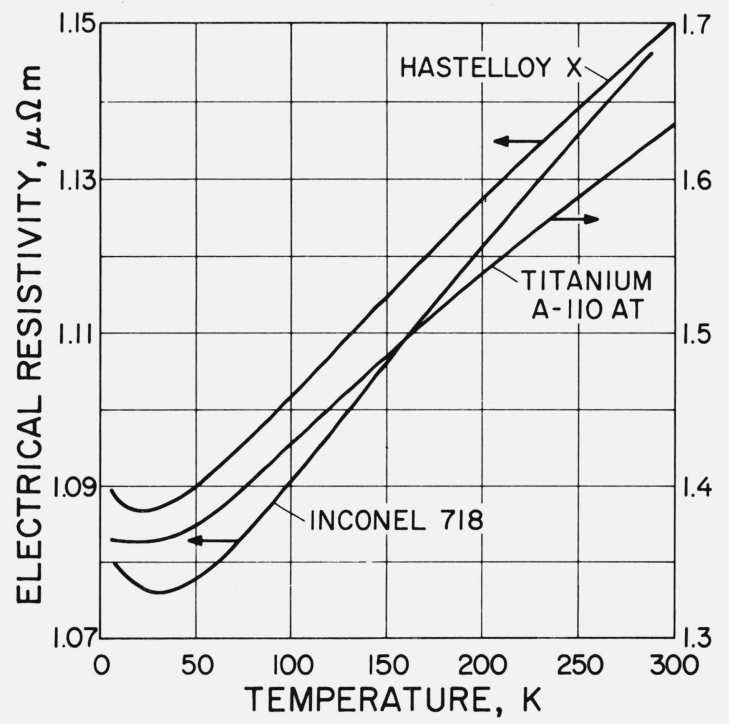

FIGURE 3. Electrical resistivity of titanium A110-AT, Inconel 718, and Hastelloy $X$.

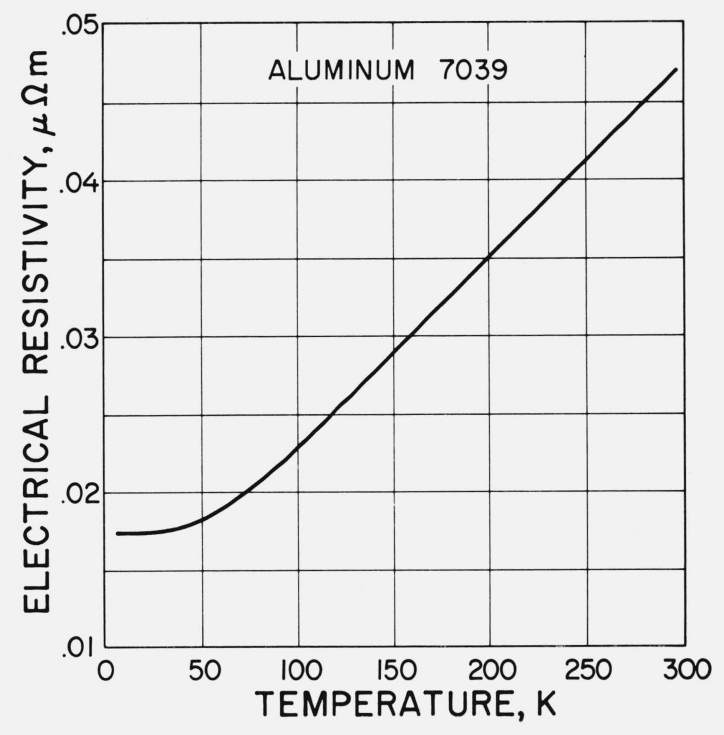

FIGURE 4. Electrical resistivity of aluminum 7039.

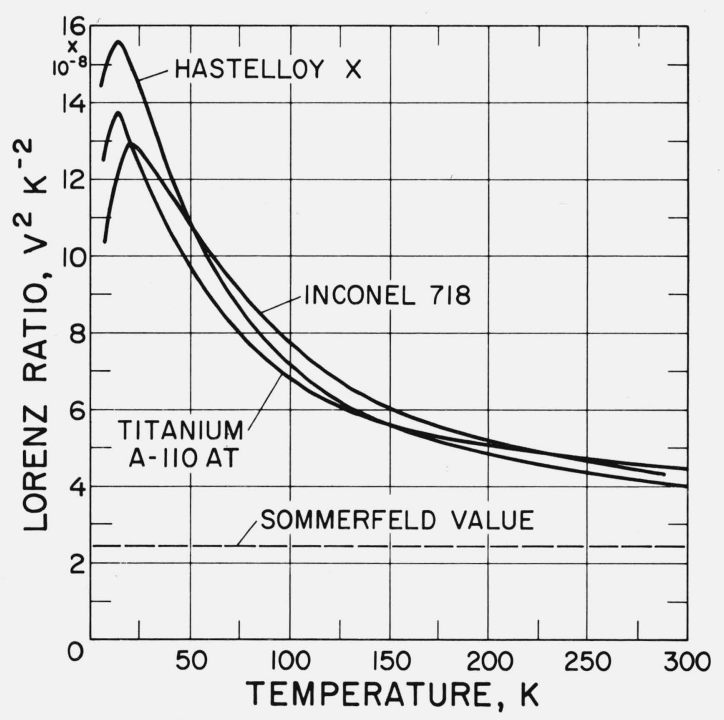

FIGURE 5. Lorenz ratios of titanium A110-AT, Inconel 718, and Hastelloy $X$. 


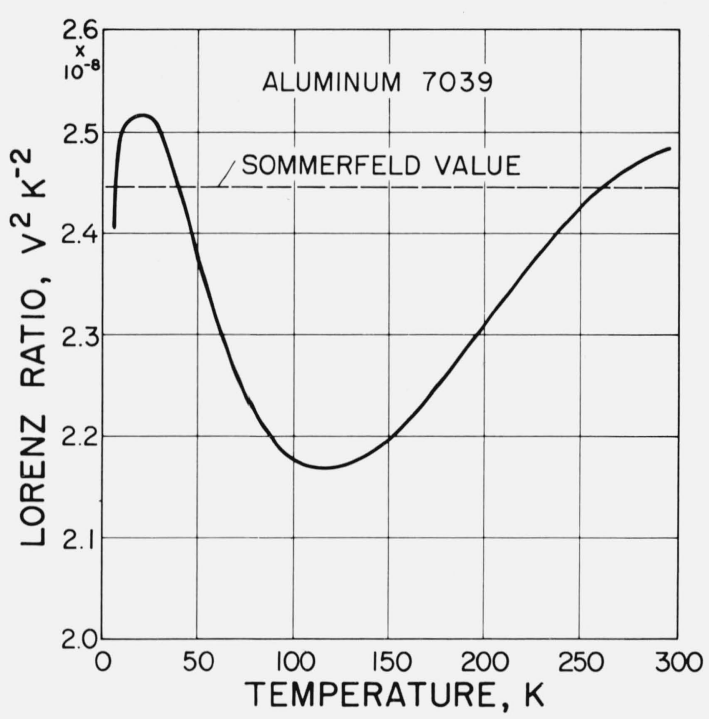

FIGURE 6. Lorenz number of aluminum 7039.

errors the uncertainty estimates (with 95\% confidence) are as follows:

thermal conductivity:

electrical resistivity: thermopower:

\author{
$2.5 \%$ at $300 \mathrm{~K}$, decreasing as \\ $\mathrm{T}^{4}$ to $0.70 \%$ at $200 \mathrm{~K}, 0.70 \%$ \\ from $200 \mathrm{~K}$ to $50 \mathrm{~K}$, increasing \\ inversely with temperature to \\ $1.5 \%$ at $4 \mathrm{~K}$. \\ $0.25 \%$ \\ $0.5 \%+0.2 \mu \mathrm{V} / \mathrm{K}$ at $4 \mathrm{~K}, 0.2 \%$ \\ $+0.05 \mu \mathrm{V} / \mathrm{K}$ at $30 \mathrm{~K}$, and \\ $0.1 \%+0.03 \mu \mathrm{V} / \mathrm{K}$ above $76 \mathrm{~K}$.
}

The thermopower values given here are absolute values although our measurements were carried out with respect to normal silver wire. The absolute thermopowers of normal silver reported by Borelius et al. [3] were used to convert the experimental data to the absolute scale.

\section{Discussion}

The titanium alloy $\mathrm{Ti}$ A110-AT and the nickel alloys Inconel 718 and Hastelloy $\mathrm{X}$ exhibit similar behavior in many respects. The thermal conductivity values are not only similar in trend but also are the same in magnitude within about 20 percent. The electrical resistivity of each of these materials is high and the resistance change from room temperature to liquid helium is only 10 to 20 percent. Each of the electrical resistivity curves exhibits a minimum near $25 \mathrm{~K}$. The high Lorenz ratios of these materials indicates that the lattice contribution to the total conductivity is up to six times as large as the electron contribution. Such high lattice contributions for alloys are often alluded to in the literature but not often confirmed experimentally.

The aluminum alloy (7039) has a thermal conductivity trend similar to the titanium and nickel alloys; however, the magnitude of the conductivity of Al 7039 is about an order of magnitude higher. Also, it is clear

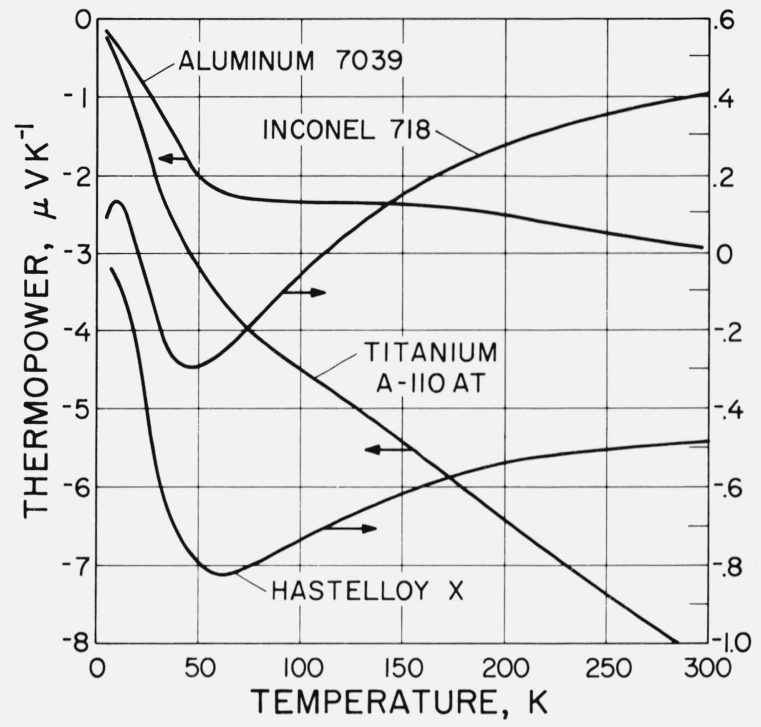

FigURE 7. Thermopower of titanium A110-AT, aluminum 7039, Inconel 718, and Hastelloy $X$.

from the Lorenz ratio that the heat conduction is primarily due to the electron contribution rather than the lattice. The electrical resistivity is smaller for Al 7039 than for the titanium and nickel alloys, and the electrical resistivity ratio is about 2.6. No minimum occurs in the electrical resistivity curve of $\mathrm{Al} 7039$.

Component analysis of the low conductivity alloys has been accomplished as follows: We assume additivity of electronic $\left(\lambda_{e}\right)$ and lattice $\left(\lambda_{g}\right)$ thermal conductivities, i.e.,

$$
\lambda=\lambda_{e}+\lambda_{g}
$$

The reciprocal of the electronic conductivity, which is the thermal resistivity, $W_{e}$, is separated into impurity $\left(W_{0}\right)$ and intrinsic $\left(W_{i}\right)$ components:

$$
\lambda_{e}^{-1} \equiv W_{e}=W_{0}+W_{i} .
$$

The electrical resistivity can be similarly separated:

$$
\rho=\rho_{0}+\rho_{i} .
$$

The impurity component, $W_{0}$, is computed from the Wiedemann-Franz-Lorenz law

$$
W_{0}=\frac{\rho_{0}}{L_{0} T}
$$

where $L_{0}=$ Sommerfeld value of Lorenz number and $\rho_{0}$ is the residual electrical resistivity. The intrinsic thermal resistivity term $W_{i}$ is computed from

$$
W_{i}=\frac{\rho_{i}}{L_{i} T},
$$

where $L_{i}$ is the intrinsic electronic Lorenz function and $\rho_{i}$ is the intrinsic electrical resistivity. 
The function $L_{i}$, as obtained from other measurements and theory, approximately approaches $L_{0}$ at high temperature and falls to zero quadratically at very low temperature. The general character of $L_{i}$ as a function of temperature is illustrated by White and Rosenberg [6].

Values for the intrinsic Lorenz function are obtained from data reported by White and Woods [5] for copper, sodium, and several transition elements. These data $\left(L_{i}=\rho_{i} / W_{i} T\right)$ were plotted versus $T / \theta_{D}$, where $\theta_{D}$ is the Debye temperature. An "average" curve weighted heavily toward copper was drawn and used for $L_{i}$ values in this work. There is a relatively large uncertainty here because of the spread in the curves for different materials; however, since $W_{i}$ is small compared to $W_{0}$ for low conductivity alloys, the overall error in $W_{0}$ is small. Values of $L_{i}$ versus $T / \theta_{D}$ are given in table 10. We thus obtain the electronic thermal conductivity component

$$
\lambda_{e}=W_{e}^{-1}=\left(\frac{\rho_{0}}{L_{0} T}+\frac{\rho-\rho_{0}}{L_{i} T}\right)^{-1} .
$$

We then obtain the lattice thermal conductivity component from (4) and (8)

$$
\lambda_{g}=\lambda-\left(\frac{\rho_{0}}{L_{0} T}+\frac{\rho-\rho_{0}}{L_{i} T}\right)^{-1}
$$

TABLE 10. Intrinsic Lorenz Ratios

\begin{tabular}{l|l}
\hline \hline & \multicolumn{1}{|c}{$L_{i}$} \\
$T / \theta_{D}$ & \multicolumn{1}{|c}{$V^{2} / K^{2}$} \\
\hline 0 & $0 \times 10^{-8}$ \\
0.05 & 0.32 \\
.10 & .80 \\
.15 & 1.12 \\
.20 & 1.35 \\
& \\
.25 & 1.53 \\
.30 & 1.69 \\
.35 & 1.81 \\
.40 & 1.92 \\
.45 & 2.02 \\
& \\
.50 & 2.10 \\
.60 & 2.23 \\
.70 & 2.32 \\
.80 & 2.37 \\
.90 & 2.40 \\
1.00 & 2.42 \\
1.50 & 2.43 \\
2.00 & 2.44 \\
\hline
\end{tabular}

The values of $\lambda_{g}$ calculated from (9) are shown in figure 8. At low temperatures, $\lambda_{g}$ varies as $T^{n}$ where $1<n \leqslant 2$. A value of 2 for $n$ is expected for phonon scattering by dislocations and electrons. Since $n$ is not equal to 2 at the lowest temperatures measured, there probably is considerable planar defect scattering, due to stacking faults or laminar precipitates at the grain boundaries. For planar defect scattering $n=1$ is expected. At higher temperatures the behavior of the curve is consistent with point imperfection scattering of the lattice waves.

Preliminary calculations indicate that the lattice conductivity of Al 7039 is comparable to the uncertainty in the measured conductivity plus the uncertainty in the calculated value of $\lambda_{e}$; therefore, these lattice conductivity values are not presented.

At this time it is difficult to be sure of which scattering mechanisms are present, especially at the lower temperatures. Further metallurgical investigations on these specimens may lead to a better understanding of the mechanisms present.

We thank R. H. Kropschot for his many helpful suggestions, discussions, and guidance. The supply of materials from Aerojet General Corporation, Titanium Corporation of America, and Union Carbide Co. is also acknowledged. The assistance of R. P. Reed and R. L. Durcholz on material characterization is appreciated. Of great help was the instrumentation design and development by J. C. Jellison and N. C. Winchester. G. H. Wallace assisted in the early phases of apparatus construction. William J. Hall made available his orthonormal fitting routine, which has been helpful in fitting the data.

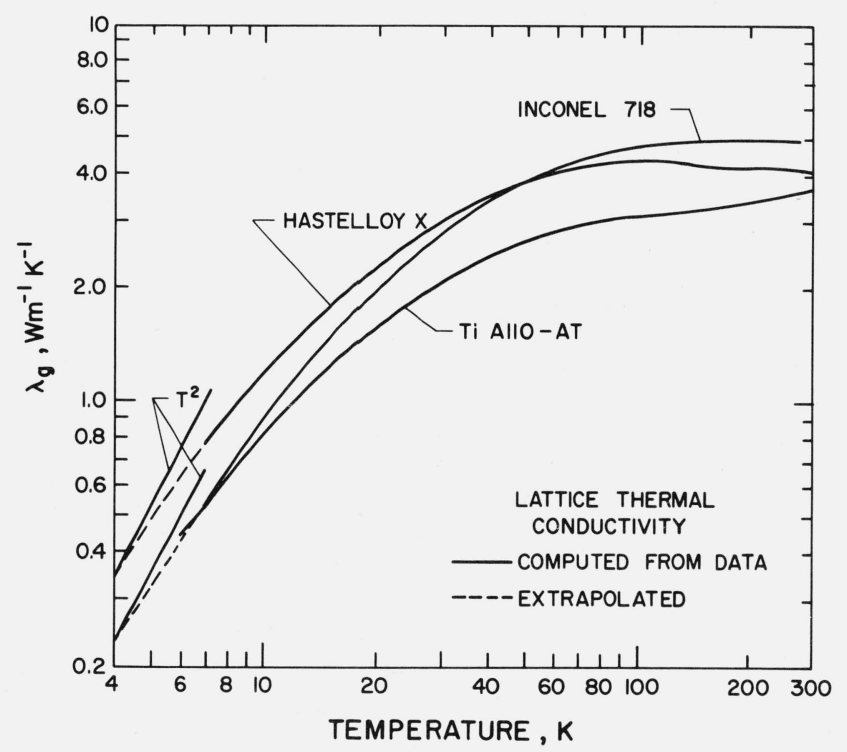

FIGURE 8. Lattice conductivity of titanium A110-AT, Inconel 718, and Hastelloy $X$. 


\section{References}

[1] Powell, R. L., Rogers, W. M., and Coffin, D. O., J. Res. Nat. Bur. Stand. (U.S.) 59 (Phys. and Chem.), 349-355 (1957).

[2] Hust, J. G., Powell, R. L., and Weitzel, D. H., J. Res. Nat. Bur. Stand. (U.S.) 74A (Phys. and Chem.), No. 5, 673-690 (Sept.Oct. 1970).

[3] Borelius, G., Keesom W. H., Johansson, C. H., and Linde, J. O., Proc. Kon. Akad. Amsterdam 35, 10 (1932).

[4] Powell, R. L., Roder, H. M., and Hall, W. J., Phys. Rev. 115, 314-323 (1959).

[5] White, G. K., Proc. of 8th Conf. on Thermal Conductivity, Eds. Ho, C. Y., Taylor, R. E., (Plenum Press, New York, 1969).
[6] Rosenberg, H. M., Low Temperature Solid State Physics Clarendon Press, Oxford, 1963). p. 120.

[7] White, G. K., and Woods, S. B., Phil. Trans. Roy. Soc. A251, 273-302 (1959).

[8] Hastelloy-X is a registered trade name for a nickel-chromium-iron alloy produced by Union Carbide Corporation, Stellite Division. Inconel-718 is a registered trade name for a nickel-chromiumiron alloy produced by International Nickel Corp. Chromel is a registered trade name of a thermocouple alloy produced by Hoskins Manufacturing Co.

(Paper 75A4-668) 